\title{
INSIGHTS
}

\section{Three months of COVID-19 in a pediatric setting in the center of Milan}

\author{
Carlo Agostoni $^{1,2}$, Giuseppe Bertolozzi ${ }^{1}$, Barbara Cantoni ${ }^{1,3}$, Carla Colombo ${ }^{1,2}$, Giovanni Montini ${ }^{1,2}$ and Paola Marchisio $^{1,2}$
}

\begin{abstract}
The second epicenter of the global COVID-19 epidemic following Wuhan, and the first in the Western world, occurred unexpectedly in the Lombardy region of Italy, whose capital city is Milan. The aggressive nature of the outbreak in the region was dramatic, leading to a 2-month period of lockdown. Within the Policlinico, the historic hospital in the center of Milan, many units were rapidly converted into intensive care units or semi-intensive units for adult patients. During lockdown, the pediatric inpatient units had to face daily reorganization caused by the necessary logistic and structural transformations, thus restricting routine care pathways for chronic patients, while the Pediatric Emergency Unit had to develop a system able to effectively separate the children and caregivers infected with COVID-19 from those who were not affected. These 2 months enhanced resilience among both doctors and nurses, and facilitated the transversal transmission of data aimed at helping colleagues and patients in any way possible, in spite of the restrictive measures limiting the rate of activity in pediatric care. The reorganization of the current phase of decreasing epidemic activity still leaves us with unanswered questions regarding the further possible changes to implement in the event of a potential reoccurrence of epidemic peaks.
\end{abstract}

Pediatric Research (2021) 89:1572-1577; https://doi.org/10.1038/s41390-020-01108-8

\section{INTRODUCTION: FOCUS ON THE CONTEXT}

At the end of February 2020, the COVID-19 pandemic took firm hold in the north of Italy, and from there it seemed that the ongoing global spread of the virus spiralled out of control. Among the eight regions of Northern Italy, Lombardy (10 million inhabitants) currently has the highest number of COVID diagnoses, accounting for one in three of all nationwide infections and one in two deaths. With $\sim 16,000$ deaths recorded by the end of May, the Lombardy region ranked sixth among the countries of the world for COVID-19-related deaths, yet in spite of this period of understandably negative growth rates (website www.truenumbers.it updated May 26), it is still responsible for $>20 \%$ of the Italian gross domestic product. Milan is the capital city of Lombardy and the largest in terms of population density, and it is widely recognized as the economic center of Italy. Although Milan was initially indirectly affected by the pandemic, as the most affected towns were situated to the south and east of the city (the Italian patient zero was from Codogno, to the south, while Bergamo and Brescia lie to the east), this soon changed for the worse.

At the end of March, when I (C.A.) was first asked by the Editors of Pediatric Research to report on the COVID situation in Milan, we felt like the Israelites fleeing Egypt, crossing the Red Sea, with towering walls of seawater on either side of us, the Egyptian army and the Pharaoh behind, and the Promised Land lying somewhere over the horizon, out of sight. What was unfolding and which direction it would take seemed to be in the hands of the Fates. "Andrà tutto bene," "Everything will be OK," were the words of hope we could read on every corner of a deserted, locked-down Milan; and for what felt like an eternity, we lived in hope of finally finding a Promised Land that nobody could envisage. Milan was cold and deserted during those days. Figure 1 contains a series of photos taken at 3 p.m. on March 30, which show a deserted Duomo Square in Milan's Old Town and empty streets in the famous Brera Fashion District.

In our hospital, IRCCS Policlinico, the oldest and largest hospital in the center of Milan, and one of the of the most important in the region, the pediatric building (The De Marchi Clinic) was literally surrounded by adult units, which were being rapidly converted into either intensive care units (ICUs) for critical patients or clinical units for the less severe cases, all COVID-19 positive. Fortunately for us, the core of the clinical emergency did not involve children, even those who were theoretically more at risk, such as, for instance, primary and secondary immunosuppressed patients (kidney transplant recipients, rheumatological cases, primary immunodeficiencies, autoimmune liver disorders, and some inborn errors of metabolism) or especially fragile groups of patients, such as those with cystic fibrosis (CF). At the same time, our good friend Lorenzo D'Antiga, Director of the Pediatric Unit at the Giovanni XXIII Hospital in Bergamo, one of the worst affected cities, was asked to provide voluntary support to the COVID-19 adult units due to the limitation of resources caused by this unprecedented, unexpected, and tragic situation. ${ }^{1}$ We hereby report the changes in clinical activity, the unanswered questions, and the pragmatic solutions, as well as the changes in the didactic and scientific activities, that the Directors of each individual Pediatric Unit in the Pediatric Area of the IRCCS Policlinico of Milan had to deal with. Of note, the few of us who contracted COVID-19 went into mandatory quarantine, while those with milder forms continued to work since swab testing was compulsory only in the case of ascertained fever.

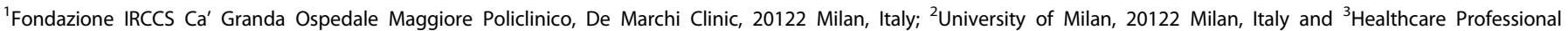
Department, 20122 Milan, Italy

Correspondence: Carlo Agostoni (carlo.agostoni@unimi.it)

Received: 20 July 2020 Accepted: 26 July 2020

Published online: 27 August 2020 


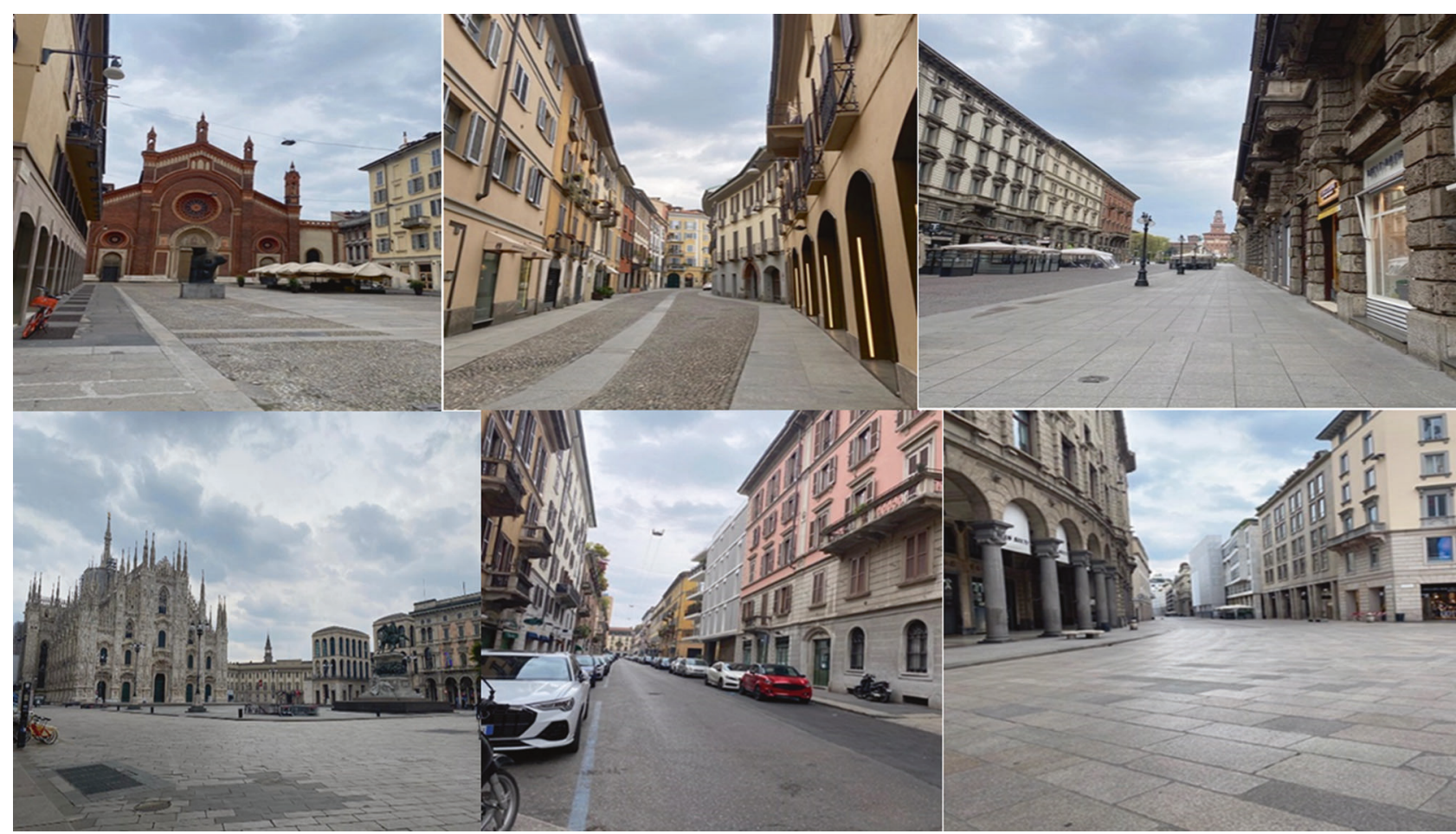

Fig. 1 The empty center of Milan, March 30, 2020, 3 p.m. Panels (clockwise) represent the "Milano Brera fashion district" (1,2,3,4,5) and Duomo Square (6), respectively.

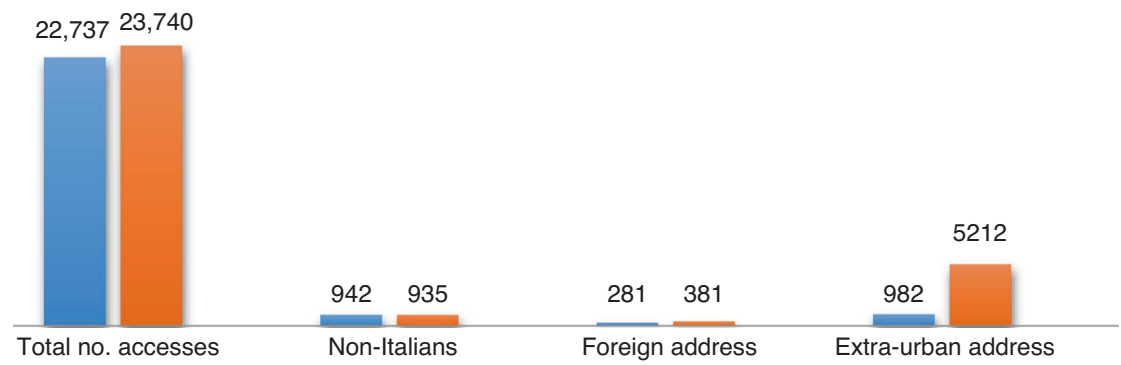

Fig. 2 Number of accesses to the Pediatric Emergency Unit, IRCCS Policlinico, Milan, for the years 2018 (blue bar) and 2019 (red bar).

\section{PEDIATRIC EMERGENCY UNIT (PEU): THE MIXED AREA}

The PEU of the hospital is considered to be a major referral center for pediatric emergencies by the citizens of Milan, as illustrated in Fig. 2, which reports the total number of accesses recorded for the years 2018 and 2019. The PEU has two exam rooms, a resuscitation room for the initial stabilization of critically ill patients, and four beds for short-stay observation. At the onset of the pandemic, the unit was subjected to some extremely stressful changes that were necessary for the real-time, mandatory planning of a system for the identification of two structurally welldifferentiated pathways for suspected and non-suspected cases, respectively, with the aim of preventing any contact between these two groups of patients and thus the possible spread of the infection. It must be said that during the days of mourning that followed, it became very difficult for all of us to separate potentially infected patients from those for whom infection seemed unlikely. Indeed, on the basis of the data available, two dedicated areas were initially identified. These were quickly increased to four, due to the rising difficulty we experienced in separating suspected from non-suspected cases because of the rapid spread of COVID-19, and the concept, although not evidence-based, of a possible asymptomatic status in children. Within this context, the triage process became increasingly important, and a staff of nurses with advanced triage training and long-standing experience was promptly established.

The potential overcrowding of the unit was avoided because of the significant decrease in admissions immediately following the beginning of the lockdown phase. The mean number of cases presenting daily at the PEU during lockdown was $\sim 28 \%$ of those presenting during the same period of the previous year (on average 69 vs. 20 patients per day, between March 1 and May 4, in 2019 and 2020, respectively), with no significant difference seen in the distribution of patients according to gravity assessed at triage (Fig. 3).

All patients accessing the PEU were interviewed upon arrival, using an anamnestic questionnaire drafted according to the most recent data available in the literature (homemade, de facto). The main assessment criteria were: (1) recent travel to local epidemic areas, and (2) the presence of pyrexia $\left(\geq 37.5^{\circ} \mathrm{C}\right)$ and/or cough and/or shortness of breath. Following the legislative decree from the Italian government dated March 3, and the World Health Organization declaration of global pandemic issued 7 days later on March 11, the area of residence became irrelevant in terms of the classification of patients, while triage assessment criteria were expanded to include any contact the caregiver may have had with a suspected or proven case of COVID-19, together with flu-like symptoms in the caregiver. 


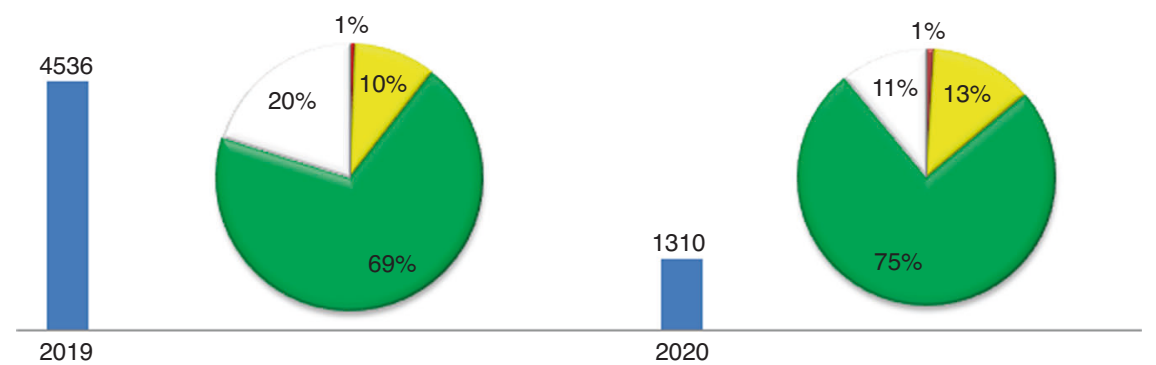

Fig. 3 Total number of accesses (blue bars) to the PEU for the period March 1 to May 4 for 2019 and 2020, according to priority codes at triage: white, green, yellow, and red, as percentages of total accesses.

Table 1. Decision-making algorithm at PEU triage.

First assessment of Emergency $\rightarrow$ Attribution of a Code Red based on vital signs or PAT $\rightarrow$

To be managed in Resuscitation Room

- Treat patient and caregiver as if they were positive for COVID-19

- Use of DPI for every team member

- Communicate unavailable Resuscitation Room to the Regional Coordinating Center

- Complete decontamination after the patient has been treated

Table 2. Proportion of positive SARS-CoV2 testing and need for admission of children accessing the PEU, from March 1 to May $31,2020$.

Number of children tested for SARS-CoV2 following triage

Number of SARS-CoV2-positive patients admitted to the 367 inpatient COVID ward

Number of SARS-CoV2-positive patients discharged by PEU $8(2 \%)$ and sent home

Children transferred to other units

${ }^{a}$ Case one was a 9-month-old infant with neurosurgical complications and case two was a suspected Kawasaki-like syndrome, transferred to the regional Center in Bergamo.

The structural layout of the Pediatric Clinic made defining differentiated pathways for the movement of patients with a suspected COVID-19 infection and their caregivers to the designated area of the clinic without contaminating the clean areas of the Emergency Department fairly challenging. The presence of a single area equipped with advanced technologies for the support of critically ill patients (Code Red priority area) made a flow/priority triage process for emergency cases mandatory, the criteria for which are reported in Table 1. All the other patients without a Code Red priority were assessed by the triage nurse on the basis of the reported questionnaire, so as to decide whether they could be positioned in a waiting area or whether immediate isolation of the patient and the caregiver was necessary. After the initial medical evaluation, those who required a period of observation within the PEU or the dedicated Pediatric Unit (see below) for further diagnostic evaluation underwent virological screening for COVID-19 infection by means of a nasopharyngeal aspirate or swab, regardless of their reason for accessing the PEU (Table 2, virological tests performed between March 1 and May 31). The caregiver (it was obligatory that the same person remained with the child for the duration of the hospital stay) also underwent screening using the same procedure.

During the first phase of the pandemic, ICUs were completely dedicated to the management of critically ill adult patients, and the number of available beds had to be increased by converting post-op recovery areas. Our pediatric ICU was also involved in the internal reorganization of the hospital and staff were reassigned to the newly created adult ICUs, which had an easily understandable negative impact on the care of critically ill children. The management of pediatric emergencies, as a matter of fact, requires specific and robust skills, which are often lacking in health workers specialized in the care of adult patients. Thus, guaranteeing adequate intensive care to COVID-free pediatric patients was very challenging, and there were at least three acknowledged cases in which intensive care support had to be provided in the PEU for longer periods of time compared to normal conditions, making the Resuscitation Room unavailable for other urgent cases.

We feel that the management of the pandemic in our PEU should be re-assessed on the basis of our recent experience, ${ }^{2}$ considering both the continuous necessity for virological screening and the possibility that we will have to face a new phase of clinical emergency in the near future. New triage models based on sound epidemiological data and which take into consideration both the sensitivity and the specificity of recorded data, along with an appropriate evaluation of cost/benefit ratios, are necessary.

\section{ACUTE CARE PEDIATRIC UNIT: THE COVID AREA SELECTED FOR THE FIRST CLINICAL EVALUATION}

The pediatric ward has 25 beds devoted to infectious diseases, including moderate to severe respiratory infections. Up to 100 infants and children are admitted every month between October and March, influenza and bronchiolitis being the most common diagnoses, together with a few cases (5-10 per month) of tuberculosis, and severe invasive diseases, such as meningitis, sepsis, and osteomyelitis.

The first child with a suspected case of COVID-19 was admitted on February 23 for a fever and because they lived in a red zone; however, they tested negative after a few days. The initial period was hectic: at the beginning we only had three beds with all the necessary characteristics for isolation. We had to learn how to use PPEs and how to protect ourselves very quickly. We were not prepared, even if we were used to dealing with children with potentially contagious diseases, such as open multidrug-resistant tuberculosis. The main problem during this period was dealing 
with reorganization, sometimes on a daily basis, and adherence to new recommendations from the hospital directors and the Regional and Central Governments. Hours and hours had to be devoted to meetings and trying to decide, quickly, what the best way to deal with the problem was.

We adopted some new rules for safeguarding our patients and protecting healthcare personnel from infection. The recent medical history of family members was carefully collected, in order to pinpoint any risk of exposure to the virus. Every child who needed to be hospitalized (for whatever reason) underwent COVID-19 testing by means of both a nasopharyngeal swab and a nasopharyngeal aspirate. Testing was performed twice, with a time span of $24 \mathrm{~h}$ between the two tests, in order to achieve an appropriate detection rate. We recorded two cases (out of 23) that tested positive the second time a nasopharyngeal swab/aspirate was performed, $24 \mathrm{~h}$ after the first negative test result. Moreover, by performing the virus test on two simultaneous specimens (swab and aspirate), we were able to increase the rate of SARSCoV-2 detection. When comparing the results obtained from both the nasopharyngeal swabs and nasopharyngeal aspirates, we noticed that the latter was more sensitive: nasopharyngeal aspirate was positive in four patients whose swab tests were negative (aged 6 months, and 7, 9, and 11 years), while only two patients with a positive swab test had a negative aspirate test (both 8 years of age).

Since an adult was required to stay with each child during hospitalization, two nasopharyngeal swabs were also collected from the relatives available to remain as caregivers. In order to reduce the risk of infection in the healthcare workers involved in patient care, we recommended that one relative (only one and always the same person) should stay with the child until the end of the hospital stay. If it was absolutely necessary for the caregiver to change, the new adult was tested twice as well. There were a few cases in which the patient's nasopharyngeal swab was negative and the parent's was positive. The collection of the aspirate in addition to the swab, the double testing, and the testing of relatives allowed us to isolate 10 "child-relative couples" who were asymptomatic carriers of COVID-19, thus considerably reducing the risk of hospital transmission to healthcare personnel.

Patients who tested positive, or whose caregivers tested positive, were isolated in a designated high-risk area that was set up especially and kept separate from the rest of the ward. Out of the original 25 beds, 13 were devoted to COVID-19-positive ${ }^{6}$ or suspected $^{7}$ patients. Children are not little adults; they need frequent monitoring and check-ups. Even if most of the children did not have a severe disease, being positive meant that they were treated as if they did. In order to optimize organization, one pediatrician and two nurses were assigned to that area, working in 8-h shifts so as to cover each $24-\mathrm{h}$ period.

\section{CHRONIC CARE PEDIATRIC UNIT: THE MANDATORY COVID-19 FREE AREA}

The chronic care pediatric ward has 24 beds and is subdivided into three sections. The percentage of occupancy is highly variable and depends on the specific needs of the patients within each specialty.

\section{Cystic fibrosis}

The CF Reference Center of the Lombardy Region has been active in the De Marchi Clinic since the early 1970s. At present, the center has $600 \mathrm{CF}$ patients in regular follow-up (450 children and 150 adults), and provides care through a multidisciplinary team, including pediatricians, dietitians, psychologists, physiotherapists, nurses, and social workers. Standards of care for CF are well established and include recommendations regarding the multidisciplinary skills required for optimal care, as well as the number of professionals in relation to the number of patients. The frequency of outpatient visits, pulmonary function testing, and microbiological assessment is also recommended in order to provide, when needed, a timely and aggressive therapeutic intervention, which is crucial to slowing the progression of the disease. $^{3}$

Since COVID-19 is a new infection and as we have very limited clinical information so far, there was concern about its implications, especially in patients with chronic diseases, who may have a higher fatality rate compared to the general population. This may be the case for $C F$, the most frequent and potentially lethal inherited disease among Caucasians, whose morbidity and mortality is mainly related to lung disease. ${ }^{4}$ In addition, the outcome of viral respiratory infections may be particularly severe in CF patients, as documented during the $2009 \mathrm{H} 1 \mathrm{~N} 1$ influenza pandemic. ${ }^{5}$ At the beginning of the Covid-19 outbreak in Lombardy, we promptly advised our patients to self-isolate at home and adopt preventive measures, such as wearing surgical facemasks and practising adequate hand hygiene, in accordance with the extraordinary measures implemented by the Italian Government to reduce transmission. ${ }^{6}$ The CF team limited its activity to emergencies, with routine appointments, including respiratory function and microbiology testing, being postponed to prevent unnecessary hospital visits and viral spread. Communication with the multidisciplinary team remained open by means of phone calls and e-mailing, and this increased significantly immediately after lockdown. We were well aware of the potentially negative impact these changes in the level of care may have had on the outcome of CF disease over time. For this reason, as in many CF Centers all over the world, we planned the implementation of telemedicine options, ${ }^{7}$ in order to preserve the CF care model and provide support for the families by means of home monitoring with hand-held spirometers and pulse oximeters, coupled with telehealth visits or phone check-ins. Home intravenous antibiotic treatment was encouraged in an attempt to limit the negative impact of this change in the modality of care.

However, it soon became clear that the incidence of COVID-19 infection in CF patients was lower than average when compared to the general population, and the patients who became infected had a better outcome than was initially predicted. Among the 600 CF patients in regular follow-up at our center, there have been only three documented COVID-19 cases so far, all coming from highly endemic areas, and all with a mild, uncomplicated form of the disease. This is in line with the reported outcome of COVID-19 infection in $40 \mathrm{CF}$ patients (11 of whom post lung transplant) reported across eight countries through national CF registries: ${ }^{8}$ in this series with a net adult predominance (median age of 33 years and only one patient younger than 16 years), there were no fatalities, 13 (33\%) required oxygen, 1 invasive ventilation support, and $4(10 \%)$ required admission to an ICU. Of note, CF patients are quite used to segregation strategies and to the use of protective measures to prevent cross infection from dangerous respiratory pathogens. ${ }^{9}$ Certainly, there has been a great effort to avoid infection on their part and this attitude may have protected them from this very contagious virus. Alternative factors may include the frequent use of azithromycin as an anti-inflammatory therapy in this patient population and also factors related to the basic CF defect that are presently under investigation.

Nephrology, Dialysis, and Transplant Unit

The Pediatric Nephrology, Dialysis, and Transplant unit is one of the reference centers for pediatric kidney diseases in Italy and the only pediatric dialysis and kidney transplant center in the Lombardy Region. From the start of the pandemic in our area, the main concern we had for our patients was the fact that a sizeable proportion of them are considered to be fragile and at risk of any kind of infection. These patients include children on chronic dialysis, transplant recipients, and those with glomerulopathies requiring immunosuppressive drugs. These patients 
require frequent visits to our unit and, especially in the case of the children on dialysis, who have to access the hospital three times a week, are therefore at greater risk of exposure from the hospital setting and also from each other. Many of our patients have comorbidities, a high percentage has hypertension and/or cardiovascular complications, recognized risk factors for COVID19 , and some are very young children. The dramatic situation seen in adult nephrology units, who lost a startling number of transplant recipients and patients on dialysis, and whose infection rates were very high, also gave us reason for concern. ${ }^{10,11}$ Thus, in order to drastically reduce the risk of exposure to the virus in our unit, we postponed any routine clinical visits and set up a dedicated phone line that parents could contact if necessary. We organized a triage system in order to guarantee as best possible a clean environment for those entering the unit, either for dialysis or any other essential treatment. Our dialysis ward has also been totally reorganized with the aim of providing a safer environment for both patients and staff. Staff rosters have been planned to make sure that only a certain number of doctors and nurses are present in the unit at any one time, to safeguard against multiple infections among staff members.

The difficulty involved in accessing the clinics during the lockdown period and the fact that families were fearful of coming to the hospital meant that many children's conditions were left unchecked. We are now starting to see some children whose renal function has deteriorated over these 3 months of reduced ambulatory activity. In order to gain a better understanding of the actual risk for immunosuppressed children, the Italian Society for Pediatric Nephrology designed a study aimed at gathering data on the prevalence of COVID-19 infection among immunosuppressed patients and their families [data submitted]. This country's representative survey revealed that children, even if affected by advanced chronic kidney disease or under immunosuppressive therapy, did not develop clinically evident COVID-19. The European Rare Kidney Diseases Network also performed a similar study and the results of both were very promising as very few children had apparently been affected. ${ }^{12}$

\section{Chronic inflammatory disorders}

The unit for chronic inflammatory disorders offers specialized care for rheumatologic disorders, which accounts for $50 \%$ of its activity, either on the ward or in outpatient clinics. The other subspecialties include immune deficiencies (primary and/or secondary), liver disorders (a wide spectrum of disorders ranging from genetic to metabolic, and from infectious to autoimmune), food-related disorders (from allergies to chronic inflammatory intestinal diseases), and a miscellanea of other pediatric disorders mainly involving neurologic cases. Considering the immune-depressed status of most patients undergoing therapies based on drugs modulating the inflammatory response, the same concerns were raised as those previously expressed for patients with chronic kidney diseases. Besides the complexities related to the doubletest virological screening of the admitted patients and caregivers, some peculiar consequences of the COVID-19 epidemic are worth mentioning.

On the one hand, the activity of the pediatric rheumatologists was severely limited by the daily reorganization of the hospital. Anesthetists provide essential support during the invasive diagnostic and therapeutic intra-articular procedures that our group performs in children with juvenile arthritis. These activities were suddenly and greatly affected by the directives ordering the conversion of most clinical departments into ICUs for adult COVID19 patients and the related transfer of human resources. As a result of this, and also due to the reduction in outpatient activities during lockdown, invasive procedures were either cancelled or postponed; the very limited availability of our anesthetists and the reduced number of functioning operating theaters (mostly converted into ICU units) also played a part.
On the other hand, pediatric rheumatologists became a further resource for the initial, tentative treatment of adult COVID cases. When it first came to light that the biochemical picture of the worst affected COVID-19 patients resembled a cytokine storm, and not just an interstitial pneumonia mostly requiring mechanical ventilation, a virtual clinical task force transversally uniting adult and pediatric specialists and including infectious diseases specialists, virologists, specialists in lung diseases, rheumatologists, and hematologists was created, together with anesthetists, in order to plan individualized approaches for the worst adult cases in the ICUs and to identify multilevel interventions. Pediatric rheumatologists recognized that the clinical picture of the first patients might possibly benefit from the use of cytokine blocking agents. The interleukin-1 receptor antagonist, anakinra, was chosen to be administered together with methylprednisolone, its short biological half-life being of benefit in the event of adverse reactions or secondary infections. ${ }^{13}$ As further proof of anakinra safety, children with rheumatologic disorders treated with biologic drugs (including anakinra) did not show any severe complications from COVID-19 infections. ${ }^{14}$ At the peak of the epidemic in our hospital, more than 100 patients entered this open protocol, and the clinical results after 4 weeks of treatment, when matched with untreated historical controls, were positive [data submitted].

The other admissions to the ward for chronic diseases only involved some neurologic cases, often presenting with seizures, who were hospitalized due to diagnostic and therapeutic urgency. When possible, hospital stays were drastically reduced and parental observation at home was recommended.

\section{TEACHING ACTIVITIES IN THE TIME OF COVID-19}

The problems related to the continuation of our didactic activities were, and still are, extremely complex. The end of February was a holiday period in Milan, due to the Carnival. Initially, we received instructions to abstain from lessons for a week, then 2 weeks, and then everything was locked down. Being professors of pediatrics, we were used to talking with students and residents, and to being very active in terms of giving presentations involving a lot of active discussion. Abruptly, everything stopped. And we had to learn how to use new means of communication, virtual teaching, and new platforms: this was not easy at all, especially on top of the extra clinical and organizational work.

At the University of Milan, we tried to effectively manage a great number of students, and organizing their lessons by means of an online platform in such a short space of time was extremely difficult. The situation was complicated further by the fact that some lecturers were unable to give lessons during the height of the pandemic due to increased workloads in COVID units. It was also impossible to carry out practical lessons on the wards, which obviously left students at a disadvantage in terms of their learning experience, as well as the lack of personal contact with the teachers, which is an essential part of the teacher-student relationship. Sitting exams online was also not ideal, although not impossible. On the one hand, the marked drop in clinical activity during this period meant that we were able to concentrate on research projects that were in the development phase and therefore we moved forward with these. It was also possible to complete the writing of a number of research papers. However, this reduction in clinical activity also meant that clinical trials had to be temporarily put on hold.

\section{FINAL REMARKS}

A synopsis of the situation in our hospital, mid-June, is as follows: at the height of lockdown, of the 800 beds available for inpatient admissions, 71 were switched to COVID ICU beds (bringing the total up to 85 from the pre-existing number of 14 adult ICU beds), 80 were switched to semi-intensive ICU beds, and 200 (including a 
total of 15 beds in the Pediatric Units, 2 in the PEU, and 13 in the dedicated COVID area) were allocated to mild to medium COVID cases requiring clinical observation and follow-up, that is, $>40 \%$ of the clinical resources. On the whole, 950 COVID patients have been admitted so far, with a mortality rate close to $17 \%$. It goes without saying that all the follow-up visits and other diagnostic and interventional activities were drastically reduced. A new ad -hoc building, Padiglione Fiera, was built to cope with the influx of patients; however, soon after its opening, the curve of the epidemic started to decline and only a few patients were admitted, arousing much controversy among the citizens of Milan.

After 3 months of this unexpected lesson in resilience, the most complicated in a long career in pediatrics, some positive, novel approaches and resources have been developed, which have possible applications in the COVID-19 era. ${ }^{15}$ We have learned to share problems and decision-making, and to compare data with our colleagues in branches of adult medicine and with healthcare workers of all levels. ${ }^{16}$ We have learned to communicate more with other specialists, such as virologists and laboratory technicians. We feel that we have become a real group with a common goal: to protect and cure our patients and, at the same time, ourselves. We have become part of the Italian registry for COVID19 and we are committed to sharing data with other groups dealing with different situations. We have learned to be more careful, and to be more responsible as regards the spread of infections. Social distancing, also on the wards, has become mandatory. Yet, we feel closer to each other and we are more dedicated to our cause. None of the pediatricians or nurses in our unit has tested positive: we are safe and healthy, even if exhausted. The problem is that we do not see an end to what, on some days, felt like a nightmare. We must still learn how to coexist with the coronavirus.

Finally, and most importantly, the experience of our pediatric rheumatologists, who shared clinical, diagnostic, and therapeutic decisions with their counterparts in adult medicine regarding the hospitalized COVID-19 patients, has reinforced our conviction that a children's hospital today makes more sense when it operates within the context of a general hospital, where common experiences and pathways may help to make effective decisions during the worst and most unexpected medical emergencies at the population level.

\section{ACKNOWLEDGEMENTS}

We thank the Pediatric Emergency Unit: Luca Castellazzi, Rosaria Celano, Emanuela D’Angelo, Laura Dell'Era, Roberta Ghilardi, Emanuela Laicini, Gregorio Paolo Milani, Nadia Mirra, Alessia Rocchi. Acute Care Pediatric Unit: Samantha Bosis, Ilaria Coro, Daniele Ghiglioni, Francesca Furlan, Sophie Guez, Mara Lelii, Francesca Menni, Donatella Milani, Francesca Patria, Walter Peves, Raffaella Pinzani, Giulietta Scuvera, Claudia Tagliabue. Chronic Care Pediatric Units: Cystic Fibrosis: Anna Brivio, Laura Claut, Fabiola Corti, Valeria Daccò, Nadia Faelli, Erica Nazzari, Rita Nobili, Maria Chiara Russo, Laura Zazzeron. Nephrology, Dialysis and Transplant Unit: Gianluigi Ardissino, Marta Brambilla, Valentina Capone, Silvia Consolo, Viviana D'Agostino, Antonio Mastrangelo, William Morello, Fabio Paglialonga, Ambra Sala, Jessica Serafinelli, Chiara Tamburello, Francesca Taroni, Sara Testa. Chronic Inflammatory Disorders:
Lucia Baselli, Antonio Boccazzi, Rosa Maria Dellepiane, Giovanni Filocamo, Stefano Lanni, Laura Maffeis, Francesca Minoia, Gabriella Nebbia, Federica Nuti, Antonella Petaccia, Marta Torcoletti. Nursing staff: Antonietta Biasuzzi, Gianluca Ghilardi, Caterina Migneco, Silvia Mazzoni, Fulvia Pagani, Maria Paola Vago for all the colleagues. Secretary staff: Lucia Bardugoni, Matteo Cantoni, Maria Luisa Favero, Cristina Vitaloni. A special thanks to Alexandra Teff who kindly provided the final English version.

\section{ADDITIONAL INFORMATION}

Competing interests: The authors declare no competing interests.

Publisher's note Springer Nature remains neutral with regard to jurisdictional claims in published maps and institutional affiliations.

\section{REFERENCES}

1. Nacoti, M. et al. At the epicenter of the Covid-19 pandemic and humanitarian crises in Italy: changing perspectives on preparation and mitigation. NEJM Catalyst https://doi.org/10.1056/CAT.20.0080 (2020).

2. Parri, M., Lenge, M. \& Buonsenso, D. Coronavirus Infection in Pediatric Emergency Departments (CONFIDENCE) Research Group. Children with COVID-19 in Pediatric Emergency Departments in Italy. N. Engl. J. Med. 383, 187-190 (2020).

3. Castellani, C. et al. ECFS best practice guidelines: the 2018 revision. J. Cyst. Fibros. 17, 153-178 (2018)

4. Bell, S. C. et al. The future of cystic fibrosis care: a global perspective. Lancet Respir. Med. 8, 65-124 (2020).

5. Colombo, C. et al. Influenza $\mathrm{A} / \mathrm{H} 1 \mathrm{~N} 1$ in patients with cystic fibrosis in Italy: a multicentre cohort study. Thorax 66, 260-261 (2011).

6. Colombo, C. et al. Impact of COVID-19 on people with cystic fibrosis. Lancet Respir. Med. 8, e35-e36 (2020).

7. Compton, M. et al. A feasibility study of urgent implementation of cystic fibrosis multidisciplinary telemedicine clinic in the face of COVID-19 pandemic: singlecenter experience. Telemed. J. E Health https://doi.org/10.1089/tmj.2020.0091 (2020).

8. Cosgriff, R. et al. A multinational report to characterise SARS-CoV-2 infection in people with cystic fibrosis. J. Cyst. Fibros. https://doi.org/10.1016/j.jcf.2020.04.012 (2020).

9. Holmes, A. et al. An epidemic of Burkholderia cepacia transmitted between patients with and without cystic fibrosis. J. Infect. Dis. 179, 1197-1205 (1999).

10. Valeri, A. M. et al. Presentation and outcomes of patients with ESKD and COVID19. J. Am. Soc. Nephrol. https://doi.org/10.1681/ASN.2020040470 (2020).

11. Cheng, Y. et al. Kidney disease is associated with in-hospital death of patients with COVID-19. Kidney Int. 97, 829-838 (2020).

12. Marlais, M. et al. The severity of COVID-19 in children on immunosuppressive medication. Lancet Child Adolesc. Health 4, e17-e18 (2020).

13. Filocamo, G. et al. Use of Anakinra in severe COVID-19: a case report. Int. J. Infect. Dis. 96, 607-609 (2020).

14. Filocamo, G. et al. Pediatric Rheumatology Group of the Milan Area. Absence of severe complications from SARS-CoV-2 infection in children with rheumatic diseases treated with biologic drugs. J. Rheumatol. https://doi.org/10.3899/ jrheum.200483 (2020).

15. Rosenberg, A. R. Cultivating deliberate resilience during the coronavirus disease 2019 pandemic. JAMA Pediatr. https://doi.org/10.1001/jamapediatrics.2020.1436 (2020).

16. Milani, G. P. et al. Frequency of Children vs Adults Carrying Severe Acute Respiratory Syndrome Coronavirus 2 Asymptomatically, in press [PLD200035 corrected proofs], JAMA Pediatr. (2020). 